\title{
Radiofrequency Ablation Versus Resection for Resectable Colorectal Liver Metastases: Time for a Randomized Trial?
}

\author{
Steven A. Curley, MD \\ Department of Surgical Oncology, University of Texas M. D. Anderson Cancer Center, 1400 Holcombe Boulevard, FC12.3058, \\ Houston, Texas 77030, USA
}

Treatment strategies for patients with stage IV colorectal cancer have changed dramatically in the last decade. Patients with colorectal cancer metastases confined to the liver have always been a fascinating group to consider biologically and for local regional treatment strategies. In the late 1980s through the 1990s, resection was performed for a select subset of patients who had resectable disease. However, a high proportion of patients had bilobar unresectable disease and were treated with either 5-fluorouracilbased systemic chemotherapy or with implanted hepatic arterial infusion pumps. The advent of the new millennium was associated with the availability of several new cytotoxic and biologic agents active in colorectal cancer. These agents have completely changed the approach to the treatment of patients with colorectal cancer liver metastases and thus have increased the complexity of the decision-making process in these patients.

In this issue of the Annals of Surgical Oncology, Mulier and colleagues have written a thorough and thoughtful treatise on the role of surgical therapy for colorectal cancer liver metastases. Starting in the mid1990 s, radiofrequency ablation was used to treat patients with unresectable primary or secondary hepatic malignancies in an attempt to completely eradicate the measurable disease. In some patients, radiofrequency ablation was the only local tumor treatment, and in others, some lesions were resected while others underwent concomitant thermal destruction. The

Received September 26, 2007; accepted October 1, 2007; published online: October 23, 2007.

Address correspondence and reprint requests to: Steven A. Curley, MD; E-mail: scurley@mdanderson.org

Published by Springer Science+Business Media, LLC $\odot 2007$ The Society of Surgical Oncology, Inc. putative end point in the use of radiofrequency ablation was to increase the proportion of hepatic malignancy patients treated with curative intent. Studies on the use of radiofrequency ablation for unresectable primary and metastatic liver tumors performed in the 1990s led to approval of this treatment modality by the U.S. Food and Drug Administration in 2001.

It is important to emphasize that radiofrequency ablation was approved as a treatment for unresectable hepatic malignancies. Unfortunately, this thermal ablation modality has now been applied in the treatment of both resectable and unresectable disease without the benefit of a randomized clinical trial demonstrating survival equivalence. The absence of such a clinical trial has led Mulier et al. to suggest that it may be time to perform such a study to evaluate the outcomes in patients undergoing resection versus radiofrequency ablation of otherwise resectable colorectal cancer metastases.

I enjoy a bit of controversy and debate as much as anyone, and the article by Mulier et al. should stimulate some lively discussion. Ruefully, I will state openly that I do not think it is time for a randomized clinical trial comparing resection versus radiofrequency ablation in colorectal cancer liver metastases. I have been involved in preclinical and clinical research studies that use radiofrequency ablation to treat primary and secondary hepatic malignancies since the mid-1990s. I have learned a great deal about the indications, risks, and limitations of this thermal tumor-destruction device. As a result, I now use radiofrequency ablation much less frequently in patients I treat for malignant hepatic tumors. Specifically in patients with colorectal cancer liver metastases, I now use radiofrequency ablation only to destroy one or two small tumors contralateral to the 
larger tumors that are resected. This involves $<10 \%$ of the patients with colorectal cancer liver metastases that I have treated in the past 2 years.

The reason why I believe such a prospective, randomized study would be problematic and possibly even ethically tenuous relates to a number of factors. First, the definition of what a resectable lesion is can vary dramatically from center to center on the basis of the experience and aggressiveness of the surgical team. Second, thermal ablation techniques are particularly ineffective for tumors $>5 \mathrm{~cm}$ in diameter, and this would immediately exclude patients with larger metastatic lesions. Mulier has suggested that only lesions $<3 \mathrm{~cm}$ in diameter should be included in the proposed study, but the treatment failure rate after radiofrequency ablation of even these smaller tumors is higher than local recurrence rates after definitive resection. Third, many of the colorectal cancer patients we now treat have undergone many cycles of systemic chemotherapy, leading to downsizing of their malignant hepatic lesions. An excellent response to systemic chemotherapy has increased our willingness to treat the remaining metastatic disease aggressively. Resection of the entire area of preexisting tumor is more oncologically sound than attempting thermal destruction of a frequently illdefined region in the liver. Fourth, surgical resection of colorectal cancer liver metastases when performed in high-volume centers is associated with very low surgical mortality and morbidity rates. It is unlikely that the complication rate associated with a major liver resection will ever be as low as that associated with a radiofrequency ablation, but the trade-off of definitive removal of the malignant disease compared with the uncertainty of complete destruction with the thermal ablation device is an important consideration with a value that is difficult to measure. Even in the most fastidious hands, and when performed using intraoperative ultrasonography for placement of the radiofrequency needle electrodes, incomplete destruction of the tumor with recurrence at the margins occurs at a higher rate than local recurrence along a margin-negative resection line. Of much greater concern is the fact that when radiofrequency ablation is performed transcutaneously with computed tomography or ultrasound guidance, the rate of incomplete tumor destruction can range from $10 \%$ to $>40 \%$, as reported in the radiology literature. This is a wholly unacceptable rate of treatment failure and is associated with factors that include of imprecise radiofrequency needle electrode placement, variable tumor tissue density, tumor blood flow, and influence of blood flow in adjacent large hepatic vessels.
Were these authors recommending a trial of resection versus radiofrequency ablation for earlystage hepatocellular cancer, it would be much easier to endorse such a study. All of us involved in hepatobiliary tumor surgery recognize the increased risk associated with tumor resection in the cirrhotic liver. The reported lower complication rates of radiofrequency ablation of small hepatocellular cancers compared with the high risk for morbidity and mortality after resection in these patients with coexistent chronic liver injury makes such a study a more attractive option. In this case, equivalent survival associated with a far lower complication rate in patients with cirrhosis would be an important finding. However, I do not think that a noninferiority trial with a difference in 5 -year survival of $<10 \%$ would be acceptable in colorectal cancer metastasis. A $10 \%$ reduction in survival rate with radiofrequency ablation of colorectal cancer liver metastases for resectable lesions would potentially mean that thousands of patients annually would receive inadequate treatment and could die of recurrent malignant disease as a result. Attempting to justify such a trial on the basis of "evidence-based medicine" is ethically dubious at best. Radiofrequency tumor ablation devices are imperfect, and until improvements in this technology are made that assure complete tumor destruction despite tumor density, vascularity, and proximity to major intrahepatic blood vessels in $>98 \%$ of the treated tumors, thermal tumor destruction will be associated with higher rates of local treatment failure compared with resection. Even if the devices evolve and improve, it is difficult to account in a clinical trial for the training and experience of treating physicians with optimal and appropriate placement of the radiofrequency needle electrodes. Imaging accuracy and - once again - the experience of the treating physician with real-time imaging modalities adds yet another level of complexity.

My greatest fear is that should a seemingly welldesigned, carefully performed, and thoroughly analyzed clinical trial comparing transcutaneous radiofrequency ablation with resection for colorectal cancer liver metastases be performed, it would open the floodgates (sadly, already partially opened) to the occasional practitioner of thermal tumor ablation. Oncologic principles would be abased, and the outcome of patients in the real world outside the tight control and constraints of a clinical trial would be compromised. I already see an alarming number of patients with initially resectable hepatic metastases referred to me after failure of percutaneous radiofrequency ablation. Unfortunately, some of these 
patients have resectable recurrent disease, and they ultimately die of it. It is impossible to know the proportion who would have survived in the long term had they undergone resection, but I don't think we can justify this experiment. It is not yet time for a randomized clinical trial comparing resection with radiofrequency ablation for respectable colorectal cancer liver metastases. Let the debate begin. 